\title{
A new mechanism underlying thyroid autoimmunity revealed
}

An epigenetic-genetic interaction involving a noncoding single nucleotide polymorphism (SNP) in TSHR alters thymic expression of this gene and is implicated in triggering Graves disease, according to new findings.

The researchers analysed histone $\mathrm{H} 3$ lysine 4 methylation and gene expression induced by IFN- $\alpha$ (secreted during viral infections) in human thyroid cells. Genomic regions with high levels of monomethylation were overlapped with those containing SNPs associated with Graves disease. This analysis identified two SNPs, rs12101255 and rs12101261, in intron 1 of TSHR, which encodes the TSH receptor, as methylated upon IFN- $\alpha$ induction.

"Further analysis showed that one of these SNPs, rs12101261, modifies a binding site for the transcription factor promyelocytic leukemia zinc finger protein (PLZF)," explains researcher Yaron Tomer. This analysis suggested that the allele associated with Graves disease enables binding of PLZF, which decreases expression of TSHR. Thus, the region containing this SNP is involved in regulating expression of TSHR. Expression was further decreased in response to IFN- $\alpha$. "We concluded that genetic-epigenetic interactions between IFN- $\alpha$ and the TSHR gene could predispose to Graves disease," says Tomer.

These findings suggest that epigeneticgenetic interactions are involved in many complex diseases. Tomer and co-workers are therefore planning to map all the epigenetic-genetic interactions triggered by IFN- $\alpha$ in genes associated with autoimmune thyroid diseases.

\section{Claire Greenhill}

Original article Stefan, M. et al. Genetic-epigenetic dysregulation of thymic TSH receptor gene expression triggers thyroid autoimmunity. Proc. Nat/ Acad. Sci. USA doi:10.1073/pnas.1408821111 